\title{
Application Research on Public Security Case Information Spatio-temporal Analysis in Police Practical Teaching
}

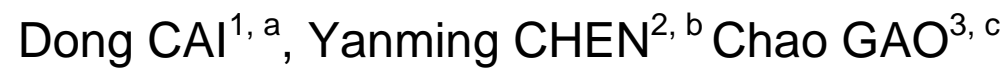 \\ ${ }^{1}$ Public Security Management Department, Jiangsu Police Institute, Nanjing, 210012, China \\ ${ }^{2}$ School of Geographic and Oceanographic Sciences, Nanjing University, Nanjing, 210004, China \\ ${ }^{3}$ Key Laboratory of Geographic Information Technology of Public Security Ministry, Changzhou \\ City Public Security Bureau, Changzhou, 213000, China \\ aemail: jickie_cd@126.com, bemail: yanming-chen @163.com, cemail: gaochao1980@163.com
}

Keywords: Case Information Spatio-temporal Analysis; Wisdom Public Security; Police Practical Teaching

\begin{abstract}
From the perspective of the wisdom public security, this paper concludes the significance of case information Spatio-temporal analysis technology and points its important position in police practical teaching. Combined with the case information Spatio-temporal analysis examples researched in emergency command teaching laboratory of public security administration in JiangSu Police institute, this paper introduces mainly functions and the typical application of case information Spatio-temporal analysis system. Then according to the public security information practices analysis, this paper concludes the common case information Spatio-temporal analysis application problems in police practical teaching, and then puts forward a tentative idea to solve the above problems.
\end{abstract}

\section{The concept of wisdom public security}

While the modern people enjoying the convenience of the city, we can understand that our city has entered a high dangerous period, traditional and non-traditional public security cases have become more frequent. The appearance of new characters of city public security, such as frequent, harm, mass, chain, and the new types of public security case make the traditional public security management mode facing severe challenges[1].

In such times background, the concept of wisdom urban comes into being, then the concept of wisdom public security comes from this concept[2]. CUDI, that is China International City Development Research Institute, proposes that the overall goal of wisdom public security is to maintain the city stability, ensure the city operation safe, and wisdom emergency management[3]. There are four aspects of wisdom public security system, including a covering a wide range cases early warning system with sensitivity response, an emergency rescue system for cooperative operation, a resource sharing information system; and an integrated collaborative organization system. The overall goal of wisdom public security greatly depends on the police geographic information system development.

\section{The significance of case information Spatio-temporal analysis application in the police practical teaching}

The case information Spatio-temporal analysis technology is an once again application of police geographic information system in public security information[4]. The police geographic information system is on the basis of public security information network and geographical information technology, and takes the police electronic map as the core. The police geographic information system provides collection, storage, management, analysis and application and expression methods for police spatial data, which can provide decision support for the police public security. The case information Spatio-temporal analysis technology is not only one of the most important modules in police geographic information system, but also is the further application of spatial analysis functions in police geographic information. 
With the rapid development of urban modernization, the traditional human knowledge of geography environment or the command on the basis of ordinary map is far from the actual requirements of public security practices. From the perspective of public security practice, on the basis of police geographic information analysis technology, introduction of case information Spatio-temporal analysis into public security can better solve the case analysis problems, such as "where", "what", and "how to deploy the police force most reasonable" and so on. Command center, criminal investigation, public security, transportation, fire, safekeeping, counter-terrorism and other departments all need comprehensive utilization of relevant business information systems superimposed on geographic information. On the basis of the above fusion data, these departments can carry out a large number of applications, such as police force dispatch and assistant decision-making, community police management and safekeeping, emergency preparedness establishment, major cases and crimes analysis, intelligent traffic management, police information resource management and thematic mapping and etc. All these applications can improve the public security practical ability to deal with illegal crime[5][6].

From the point of view of subjects of public security development, improving police geographic information system is an inevitable choice to meet the urgent need of public security emergency management personnel. The public security organ is one of the earliest government departments to use the information technology, and has accumulated rich experience in dealing with all kinds of public security cases. In this context, the public security colleges should promote the theory and conclusion of public security information practice, which is important basis of public security colleges discipline construction. Secondly, the development of case information Spatio-temporal analysis technology is the inherent need to enrich the connotation construction of public security colleges. Connotation construction is the core of discipline construction in public security colleges, and as the first level discipline, the discipline construction of public security is increasingly expanding. So recently public security colleges set up some courses about police geographic information system, and increase practical teaching contents, which can optimize specialty layout in public security colleges, and also gradually increase talent training in key areas. Thirdly, personnel in emergency management major should have strong crisis early warning and emergency response analysis, identification and treatment ability, master emergency command, executive, security technologies and etc. All these abilities can adapt to the contemporary needs of the public security work.

Therefore, with the continuous improvement of the public security information infrastructure, the application of information technology becomes deeply. Especially the construction demand of flat service command system and Macroscopic Intelligence system make it becomes urgent needs and inevitable direction of the wisdom public security development.

\section{The application of case information Spatio-temporal analysis in practical teaching}

This paper takes the construction of emergency command system in JiangSu Police institute as an example, which can be called case information Spatio-temporal analysis technology teaching system, summarizes and introduces the application of case information Spatio-temporal analysis technology in the police practical teaching. The construction target of public security case information spatio-temporal analysis system aims to carry out the case information Spatio-temporal analysis application in dispatch manage, response and control, information analysis, and crime prediction, which is based on the using of advanced technology such as computer, remote sensing, geographical information, satellite positioning and etc. On the basis of system above, JiangSu Police institute can actively participate in an application demonstration of a new generation PGIS platform, which can set up in line with the actual needs of wisdom emergency management solutions, and form the new police spatial information analysis technology with high promotion value.

The function module of case information Spatio-temporal analysis technology teaching system includes police geographic information application module, police force control application module, the entrance and exit with defense and inspection facilities application module and case analysis 
application module. Specifically, first, police geographic information application module includes display, query, zoom, query and other basic functions. Second, police force control application module can show the police force distribution situation in any time and any area. Third, the entrance and exit with defense and inspection facilities application module can realize entrance and exit information management and video monitoring function. Forth, case analysis application module can carry out a comprehensive analysis and treatment based on Classification management of police case.

The core module of case information Spatio-temporal analysis technology teaching system is the case analysis module, which can make full use of the response alarm and strike prevention and control case information to carry on some comprehensive analysis and processing in the map. All these analysis and processing is according to alarm and case classification. And after these analysis and processing, the system can find spatial and temporal distribution characteristics, which will provide intuitive analysis results for emergency command and leadership decision. Its functions are as follows:

(1)Police Intelligence Query. The case information Spatio-temporal analysis technology teaching system can provide police intelligence query according to the alarm people, the alarm telephone number, the alarm type and other information keywords, and also show the distribution of a certain kind of alarm on the map. Then according to the focus information in each specific stage, the case information Spatio-temporal analysis technology teaching system can provide flexible custom mode to study alarming information. For example, the drug trafficking and drug abuse can be combined to drug related cases, then this medium class cases will be used to subsequent analysis.

(2)High Risk Analysis. In the information Spatio-temporal analysis technology teaching system, the chosen alarm query terms can be comprehensive used to analysis the alarm high incidence time, type and area. That is, by the Spatio-temporal analysis technology, the system can research the alarm high incidence time, type and area according to the information collected by the system.

(3)Hypsometric Alarm. In the case information Spatio-temporal analysis technology teaching system, the different risk coefficient is defined by different color, which is according to Spatio-temporal analysis of the police, the amount of cases, the inflow of focus personnel, or the chain analysis, etc. Then the system can layout early warning thematic map in hypsometric color. The core function lies in the definition of risk factors, and the business department should formulate an integral rules of a set of above related factors.

(4) Crime Hotspots Analysis. When the police cases happen, the information about police case, such as case point coordinate, area and type should be input into. Then the crime hotspots, where legal case concentrated presence, could be showed and analyzed.

\section{Conclusion}

Public Security Management Department of Jiangsu Police Institute has integrated the application of information of police service into the teaching process and business model, purpose to further improve the effectiveness of practical application. The application research of public security case information spatio-temporal analysis can provide reference and decision support for the practical work in crime investigation. These research findings will put practice in the public security organs of the information department, and information department.

\section{Acknowledgement}

This work was financially supported by the Priority Academic Program Development of Jiangsu Higher Education Institutions, the Natural Science Foundation of Jiangsu Province No. BK20141033, the Theory and the Soft Science Project of Ministry of Public Security No. 2014LLYJJSST061, the University Science Research Surface Project of Jiangsu Province No. 13KJB420002, the University Philosophy, Social Sciences Research Project of Jiangsu Province No.2014SJD194, the Research Project of Jiangsu Police Institute No.13Q18, and Teaching Research 
Project of Jiangsu Province No. 2013B06.

\section{References}

[1] http://www/ibm.com/cn/zh/.

[2] Sun Jing-Jing, Guo Can. Preliminary Analysis on the Construction of Wisdom Public Security[J]. Journal of Liaoning Administrators College of Police and Justice. 2013, (1): 83-85.

[3] Wang Jun-Lin. The Study of the Connotation and Application Service System for the Smart Police[J]. Special Subject Research, 2012, (4): 14-15.

[4] Li Man-chun, Chen Gang, Chen Zhen-jie, Shao Yi-xi. GIS Design and Implementation[M]. Beijing: Science Press , 2011. 6.

[5] Xu Fa-jian. On the application of the police affairs GIS[J]. Journal of Hubei University of Police, 2007, 3, (2): 83-87.

[6] Zhen zhe. Design and Implement of the Data Integration Functions in Poloci Geography Information System of A Certain City[D]. Beijing University of Posts and Telecommunications, 2010. 